PROCEEDINGS OF THE

AMERICAN MATHEMATICAL SOCIETY

Volume 104, Number 4, December 1988

\title{
PFA IMPLIES ALL AUTOMORPHISMS ARE TRIVIAL
}

\author{
SAHARON SHELAH AND JURIS STEPRĀNS
}

(Communicated by Thomas J. Jech)

\begin{abstract}
It is shown that PFA implies that all automorphisms of the Boolean algebra $\mathscr{P}(\omega)$ /Finite are induced by almost-permutations of $\omega$.
\end{abstract}

Introduction. In $[\mathbf{S h}]$ it was shown how the oracle chain condition can be used to construct a model where all (Boolean algebra) automorphisms of $\mathscr{P}(\omega)$ /Finite are trivial in the sense that they are induced by a function which is injective and whose domain and range are both cofinite subsets of $\omega$. Equivalently, all autohomeomorphisms of $\beta \omega-\omega$ are trivial in the sense that they are restrictions of "almost" autohomeomorphisms of $\beta \omega$. While it is true that the model is obtained by a finite support iteration of countable chain condition partial orders, it is not possible to splice all countable chain condition partial orders into the iteration since some of these might not satisfy the oracle chain condition. Consequently this method is not amenable to proving the consistency of $\mathrm{MA}\left(\aleph_{1}\right)$ and all automorphisms of $\mathscr{P}(\omega) /$ Finite are trivial. That is the purpose of this paper. Familiarity with [Sh] will be assumed.

It will be shown that PFA implies that all automorphisms of $\mathscr{P}(\omega)$ /Finite are trivial. It must be pointed out that, while the consistency of PFA implies the existence of large cardinals, they are not required for the consistency of the present result. The reason is that diamond on $\omega_{2}$ can be used to replace the reflection principle which is implicit in collapsing the continuum by countably closed forcing. Hence it is possible to begin with a model of $L$ and do a countably closed iteration which will trap all possible nontrivial automorphisms in time to destroy them.

1. In order to simplify the proof two intermediate versions of the theorem will first be obtained. An automorphism $\Phi$ of $\mathscr{P}(\omega)$ /Finite will be called somewhere trivial if there is an infinite set $X \subseteq \omega$ such that the restriction of $\Phi$ to $\mathscr{P}(X) /$ Finite is trivial. An automorphism is nowhere trivial if it is not somewhere trivial. It will first be shown how to make every automorphism of $\mathscr{P}(\omega)$ /Finite somewhere trivial. It will then be shown that countably many sets on which an automorphism is trivial have an upper bound modulo a finite set on which the automorphism is also trivial. These results will then be used to prove the main theorem without having to make the various retreats which some readers found objectionable in [Sh]. It must also be admitted that it is not known whether the first two results are intermediate theorems. For example the following question remains open.

Received by the editors June 13, 1987 and, in revised form, January 11, 1988.

1980 Mathematics Subject Classification (1985 Revision). Primary 04A20; Secondary 54A35.

The first author was partially supported by Israel-US Binational Research Fund.

The second author was partially supported by NSERC. 
QUeSTION 1. Does the fact that every automorphism of $\mathscr{P}(\omega) /$ Finite is somewhere trivial imply that every automorphism is trivial?

THEOREM 1. PFA implies that all automorphisms of $\mathscr{P}(\omega) /$ Finite are somewhere trivial.

ProOF. Suppose that $\Phi: \mathscr{P}(\omega) /$ Finite $\rightarrow \mathscr{P}(\omega) /$ Finite is a nowhere trivial automorphism. Let $\mathbf{P}$ be a countably closed forcing such that $\mathbf{P}$ forces diamond. Since no new reals are added it follows that $\Phi$ remains a nowhere trivial automorphism after forcing with P. Let $\Phi^{*}: \mathscr{P}(\omega) \rightarrow \mathscr{P}(\omega)$ be a function such that the following diagram commutes, where the vertical arrows are the canonical mappings:

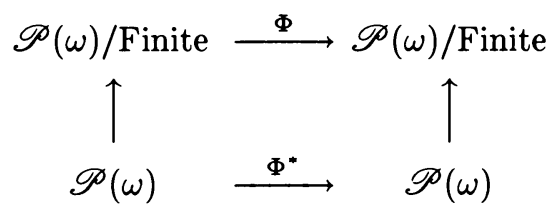

Two lemmas are required. Throughout $\equiv^{*}$ and $\not \equiv^{*}$ will refer to equivalence modulo a finite set (in other words, equivalence in $\mathscr{P}(\omega) /$ Finite).

LEMMA 1. In the extension by $\mathbf{P}$ there is a family $\left\{\left(A_{\alpha}, B_{\alpha}\right): \alpha \in \omega_{1}\right\}$ such that $\left\{A_{\alpha}: \alpha \in \omega_{1}\right\}$ is an almost disjoint family and there is a countable chain condition poset $\mathbf{Q}$ and a $\mathbf{Q}$-name $X$ such that

(1) $\mathbf{Q} \Vdash " X \cap A_{\alpha} \equiv{ }^{*} B_{\alpha}$ ".

(2) $\mathbf{Q} \Vdash “(\forall Y \subseteq \omega)\left(\exists \beta \in \omega_{1}\right)(\forall \xi>\beta)\left(Y \cap \Phi^{*}\left(A_{\xi}\right) \not \equiv^{*} \Phi^{*}\left(B_{\xi}\right)\right) "$.

ProOF. A family $\left\{\left(A_{\alpha}, B_{\alpha}\right): \alpha \in \xi\right\}=\mathfrak{A}$ will be called reasonable if $B_{\alpha} \subseteq A_{\alpha}$ for $\alpha \in \xi$ and $\pi(\mathfrak{A})=\left\{A_{\alpha}:\left(A_{\alpha}, B_{\alpha}\right) \in \mathfrak{A}\right\}$ is an almost disjoint family. If $\mathfrak{A}$ is reasonable then the partial order $\mathbf{Q}(\mathfrak{A})$ will be defined to consist of the functions $f \in{ }^{\omega} 2$ such that:

(3) $\operatorname{dom}(f) \equiv^{*} \bigcup \Sigma$ where $\Sigma \in[\pi(\mathfrak{A})]^{<\aleph_{0}}$.

(4) $f^{-1}\{1\} \cap A_{\alpha} \equiv{ }^{*} B_{\alpha}$ if $A_{\alpha} \in \Sigma$.

In $[\mathbf{S h}]$ it was shown how, given a countable reasonable family $\mathfrak{A}$, countably many sets $\left\{\mathscr{C}_{n}: n \in \omega\right\}$, each dense in $\mathbf{Q}(\mathfrak{A})$, and a $\mathbf{Q}(\mathfrak{A})$-name for a subset of $\omega$, $Y$, it is possible to find $B \subseteq A$ such that $A$ is almost disjoint from each $A_{\alpha}$ in $\pi(\mathfrak{A})$ and such that:

(5) $\mathscr{C}_{n}$ remains dense in $\mathbf{Q}(\mathfrak{A} \cup\{(A, B)\})$

(6) $\mathbf{Q}(\mathfrak{A} \cup\{(A, B)\}) \Vdash " Y \cap \Phi^{*}(A) \not \equiv * \Phi^{*}(B)$ ".

This uses the fact that $\Phi$ is nowhere trivial. There are several differences between the current presentation and that of [Sh]. First, in [Sh] an almost disjoint family $\left\{A_{\alpha}: \alpha \in \omega_{1}\right\}$ is constructed rather than a family of pairs $\left\{\left(A_{\alpha}, B_{\alpha}\right): \alpha \in \omega_{1}\right\}$. The set $X$ to which the automorphism cannot be extended almost contains certain $A_{\alpha}$ 's and is almost disjoint from others, whereas in the present discussion $X$ is constructed so that $X \cap A_{\alpha} \equiv^{*} B_{\alpha}$. However, this is really not different from what is actually being done in [Sh]; in ( $\left.{ }^{*} 1\right)$ on p. $134, P\left(\bar{A} \wedge\left\langle A, C_{l} \backslash A\right\rangle\right)$ can be replaced by $\mathbf{Q}\left(\bar{A} \cup\left(C_{l}, A\right)\right)$. Second, since the oracle chain condition is no longer being used it is not necessary to add the Cohen forcing and hence any references to this can be ignored. Finally, notice that what is really proved is not only that $\Phi$ is somewhere trivial but that if $\mathfrak{A}$ is the reasonable family constructed by the time 
the construction fails and $C$ is almost disjoint from every member of $\pi(\mathfrak{A})$ then the restriction of $\Phi$ to $C$ is trivial.

Now let $\mathscr{G}$ contain the family of all sets of functions which are candidates for being antichains in $\mathbf{Q}(\mathfrak{A})$ for some $\mathfrak{A}$ (i.e., $\mathscr{G}$ is the set of all countable sets of functions whose domain and range are contained in $\omega$ ). Since every real has a "nice" name which is a subset of $\omega \times(\mathfrak{A})$ it follows that ${ }^{\omega}[\mathscr{G}]$ contains all candidates for $\mathbf{Q}(\mathfrak{A})$-names of reals. It is therefore possible to use the continuum hypothesis to enumerate ${ }^{\omega}[\mathscr{G}]$ as $\left\{Y_{\alpha}: \alpha \in \omega_{1}\right\}$. Let $\left\{\mathscr{C}_{\alpha}: \alpha \in \omega_{1}\right\}$ be a diamond sequence. A family $\left\{\left(A_{\alpha}, B_{\alpha}\right): \alpha \in \omega_{1}\right\}$ will now be constructed inductively. Suppose that a reasonable family $\left\{\left(A_{\alpha}, B_{\alpha}\right): \alpha \in \xi\right\}=\mathfrak{A}_{\xi}$ has been constructed. Now induction on $\omega$ can be used to construct $\left\{\left(C_{n}, D_{n}\right): n \in \omega\right\}$ so that $\mathfrak{A}_{\xi} \cup\left\{\left(C_{n}, D_{n}\right): n \in k\right\}$ is a reasonable family for each $k \in \omega$ and that furthermore:

(7) If $\eta \leq \xi$ and $\mathscr{C}_{\eta}$ is a maximal antichain in $\mathbf{Q}\left(\mathfrak{A}_{\xi}\right)$ then it remains maximal in $\mathbf{Q}\left(\mathfrak{A}_{\xi} \cup\left\{\left(C_{n}, D_{n}\right): n \in k\right\}\right)$ for every $k$.

(8) $\mathbf{Q}\left(\mathfrak{A}_{\xi} \cup\left\{\left(C_{n}, D_{n}\right): n \in k\right\}\right) \Vdash$ "Y $Y_{(\Psi(k))} \cap \Phi^{*}\left(C_{k}\right) \not \equiv^{*} \Phi^{*}\left(D_{k}\right) "$ where $\Psi \in{ }^{\omega} \xi$ is a bijection and $k \in \omega$.

(9) For each $k \in \omega$ the antichains required to witness (8) remain maximal in $\mathbf{Q}\left(\mathfrak{A}_{\xi} \cup\left\{\left(C_{n}, D_{n}\right): n \in \omega\right\}\right)$.

This is easily done by using the method from [Sh] to get (5) and (6) at each stage and noting that the set of antichains mentioned in (9) is only countable.

Let $\mathbf{D}$ be the partial order for adding a dominating real (i.e. conditions in $\mathbf{D}$ are pairs $(\varphi, F)$ where $\varphi$ is a finite partial, and $F$ is a total function from $\omega$ to $\omega)$. If $G$ is the real obtained from a filter on $\mathbf{D}$ then $A_{\xi}$ will be defined to be $\bigcup\left\{C_{n} \backslash G(n)\right.$ : $n \in \omega\}$ and $B_{\xi}$ will be defined to be $\bigcup\left\{D_{n} \backslash G(n): n \in \omega\right\}$. It will not be difficult to find $G$ since it will only be required to meet countably many dense sets. For each $\varsigma \in \xi$ it is easy to see that the set of conditions in $\mathbf{D}$ forcing that $A_{\xi}$ is almost disjoint from $A_{\varsigma}$ is dense.

Since we do not yet know which $G$ will be used to define $A_{\xi}$ and $B_{\xi}$, it is not possible to say what the elements of $\mathbf{Q}\left(\mathfrak{A} \cup\left\{\left(A_{\xi}, B_{\xi}\right)\right\}\right)$ are. However each of them can be represented as $p \cup \chi \backslash h$, where $p \in \mathbf{Q}(\mathfrak{A}), \chi$ is a $\mathbf{D}$-name for the characteristic function of $B_{\xi}$ restricted to $A_{\xi}$, and $h$ is a finite function. Therefore it suffices to show that if $p \in \mathbf{Q}(\mathfrak{A}), h$ is a finite function and $\mathscr{C}$ is a maximal antichain in $\mathbf{Q}\left(\mathfrak{A}_{\xi} \cup\left\{\left(C_{n}, D_{n}\right): n \in k\right\}\right)$ then $\{(\varphi, F) \in \mathbf{D}:(\exists q \in \mathscr{C})(\varphi, F) \Vdash " q$ and $p \cup \chi \backslash h$ are compatible" $\}$ is dense in $\mathbf{D}$. To see that this suffices, note that for each $k \in \omega$ and $m \in \omega$ there is a maximal antichain contained in

$$
\begin{aligned}
& \left\{p \in \mathbf{Q}\left(\mathfrak{A}_{\xi} \cup\left\{\left(C_{n}, D_{n}\right): n \in k\right\}\right) ;\right. \\
& \left.\quad p \Vdash " j \in\left(Y_{\boldsymbol{\Psi}(k)} \cap C_{k} \backslash D_{k}\right) \cup\left(D_{k} \backslash Y_{\Psi(k)}\right) \text { and } m \leq j "\right\} .
\end{aligned}
$$

This will ensure that (6) holds.

To prove that these sets are dense let $(\varphi, F) \in \mathbf{D}, p \in \mathbf{Q}(\mathfrak{A}), h \in \omega$ and $\mathscr{C}$ be a maximal antichain in $\mathbf{Q}\left(\mathfrak{A}_{\xi} \cup\left\{\left(C_{n}, D_{n}\right): n \in k\right\}\right)$. First extend $(\varphi, F)$ to $\left(\varphi^{\prime}, F\right)$ such that $k \in$ domain $\left(\varphi^{\prime}\right)$. Then define $\chi$ so that domain $(\chi)=\bigcup\left\{C_{n} \backslash \varphi^{\prime}(n): n \in\right.$ $\left.\operatorname{domain}\left(\varphi^{\prime}\right)\right\}$ and $\chi^{-1}\{1\}=\bigcup\left\{D_{n} \backslash \varphi^{\prime}(n): n \in\right.$ domain $\left.\left(\varphi^{\prime}\right)\right\}$. Then there is $q \in \mathscr{C}$ such that $q$ is compatible with $p \cup \chi \backslash h$. Since $q \in \mathbf{Q}\left(\mathfrak{A}_{\xi} \cup\left\{\left(C_{n}, D_{n}\right): n \in k\right\}\right)$ it follows that domain $(q) \cap C_{j}$ is finite for each $j \geq$ domain $\left(\varphi^{\prime}\right)$ and hence there is $F^{\prime}$ dominating $F$ such that domain $(q) \cap C_{j} \subseteq F^{\prime}(j)$. It now follows that $\left(\varphi^{\prime}, F^{\prime}\right) \Vdash$ " $q$ is compatible with $p \cup \chi \backslash h "$. 
If $\mathfrak{A}=\left\{\left(A_{\alpha}, B_{\alpha}\right): \alpha \in \omega_{1}\right\}$ is constructed by induction then a standard argument shows that $\mathbf{Q}(\mathfrak{A})$ has the countable chain condition. From (8) and (9) it follows that (2) holds. To see that (1) holds simply let $X=\bigcup\left\{f^{-1}\{1\}: f \in G\right\}$ where $G$ is $\mathbf{Q}(\mathfrak{A})$-generic.

LEMMA 2. $\mathbf{P} * \mathbf{Q}(\mathfrak{A})$ forces that there is a proper partial order $\mathbf{R}$ and a name $I$ such that $\mathbf{R}$ forces the following statement: $I \in\left[\omega_{1}\right]^{\aleph_{1}}$ and if $\{\alpha, \beta\} \in[I]^{2}$ then either $\left(\Phi^{*}\left(A_{\alpha}\right) \backslash \Phi^{*}\left(B_{\alpha}\right)\right) \cap \Phi^{*}\left(B_{\beta}\right) \neq 0$ or $\left(\Phi^{*}\left(A_{\beta}\right) \backslash \Phi^{*}\left(B_{\beta}\right)\right) \cap \Phi^{*}\left(B_{\alpha}\right) \neq 0$.

PROOF. Let $R$ be the relation on $\omega_{1}$ defined by $\{\alpha, \beta\} \in R$ if and only if $\left(\Phi^{*}\left(A_{\alpha}\right) \backslash \Phi^{*}\left(B_{\alpha}\right)\right) \cap \Phi^{*}\left(B_{\beta}\right) \neq 0$ or $\left(\Phi^{*}\left(A_{\beta}\right) \backslash \Phi^{*}\left(B_{\beta}\right)\right) \cap \Phi^{*}\left(B_{\alpha}\right) \neq 0$. In [ARS] a relation on $S$ is defined to be semiopen if it can be expressed as $\bigcup\left\{D_{n} \times E_{n}: n \in \omega\right\}$ where $D_{n} \cup E_{n} \subseteq S$ for each $n \in \omega$. It is easy to see that $R$ is a semiopen relation since $\{\alpha, \beta\} \in R$ can be witnessed by an integer. In [ARS] it is shown that for every semiopen relation on $\omega_{1}$ whose complement does not contain an uncountable homogeneous set, there is a proper partial order which adds an uncountable homogeneous set for the relation. Define $\mathbf{R}$ to be this partial order. The only thing to show is that the homogeneous set is not disjoint from $R$.

To see this suppose that $H \in\left[\omega_{1}\right]^{\aleph_{1}}$ is uncountable and $[H]^{2}$ is disjoint from $R$. Then let $Y=\bigcup\left\{B_{\alpha}: a \in H\right\}$. It is easily verified that $Y \cap \Phi *\left(A_{\alpha}\right)=\Phi *\left(B_{\alpha}\right)$ for each $\alpha \in H$ contradicting the choice of $\mathbf{Q}(\mathfrak{A})$.

Since $\mathbf{P} * \mathbf{Q}(\mathfrak{A}) * \mathbf{R}$ is proper and it only takes $\aleph_{1}$ dense sets to ensure that all of the conditions in Lemmas 1 and 2 are met, it follows that there is a reasonable family $\left\{\left(A_{\alpha}, B_{\alpha}\right): \alpha \in \omega_{1}\right\}$ and $X \subseteq \omega$ such that

(10) $X \cap A_{\alpha} \equiv^{*} B_{\alpha}$ for $\alpha \in \omega_{1}$.

(11) $\left(\Phi^{*}\left(A_{\alpha}\right) \backslash \Phi^{*}\left(B_{\alpha}\right)\right) \cap \Phi^{*}\left(B_{\beta}\right) \neq 0$ or $\left(\Phi^{*}\left(A_{\beta}\right) \backslash \Phi^{*}\left(B_{\beta}\right)\right) \cap \Phi^{*}\left(B_{\alpha}\right) \neq 0$ for $\{\alpha, \beta\} \in\left[\omega_{1}\right]^{2}$.

But $\Phi^{*}(X) \cap \Phi^{*}\left(A_{\alpha}\right) \equiv^{*} \Phi^{*}\left(X \cap A_{\alpha}\right) \equiv^{*} \Phi^{*}\left(B_{\alpha}\right)$ for $\alpha \in \omega_{1}$. Hence there are $m, A, B$ and $\Gamma \in\left[\omega_{1}\right]^{\aleph_{1}}$ such that if $\alpha \in \Gamma$ then $\Phi^{*}(X) \cap \Phi^{*}\left(A_{\alpha}\right) \backslash m=\Phi^{*}\left(B_{\alpha}\right) \backslash m$ and $\Phi^{*}\left(A_{\alpha}\right) \cap m=A$ and $\Phi^{*}\left(B_{\alpha}\right) \cap m=B$. Now if $\{\alpha, \beta\} \in[\Gamma]^{2}$ then it follows from (11) that it may be assumed that there is $k \in\left(\Phi^{*}\left(A_{\alpha}\right) \backslash \Phi^{*}\left(B_{\alpha}\right)\right) \cap \Phi^{*}\left(B_{\beta}\right)$. But this is impossible, since if $k<m$ then $k \in(A \backslash B) \cap B$. On the other hand if $k \geq m$ then $k \in \Phi^{*}(X)$, since $k \in \Phi^{*}\left(B_{\beta}\right)$. But then $k \in \Phi^{*}\left(B_{\alpha}\right)$, which is a contradiction.

2. Given an automorphism $\Phi$ let $T(\Phi)$ be the ideal of subsets of $\omega$ on which $\Phi$ is trivial. It has already been shown that $T(\Phi)$ is dense in $\mathscr{P}(\omega) /$ Finite. The present section is devoted to showing that, in addition, $T(\Phi)$ is a $p$-ideal.

THEOREM 2. PFA implies that if $\Phi$ is an automorphism of $\mathscr{P}(\omega) /$ Finite then $T(\Phi)$ is a nontrivial $p$-ideal.

PROOF. The proof of Theorem 2 is very similar to the proof of Theorem 1 . Let $\left\{C_{n}: n \in \omega\right\}$ be disjoint sets in $T(\Phi)$. It must be shown that there is a set $C \in T(\Phi)$ such that $C \supseteq^{*} C_{n}$ for each $n \in \omega$. The main change to the proof which must be made is that the reasonable family $\left\{\left(A_{\alpha}, B_{\alpha}\right): \alpha \in \omega_{1}\right\}$ which is constructed must satisfy the additional property that $A_{n}=C_{n}$ for $n \in \omega\left(B_{n}\right.$ can be arbitrary). The proof then proceeds exactly as in $\S 1$. This, of course, means that at some stage $\beta$, the construction of the reasonable family fails. As in $\S 1$, this means that if $D$ is almost disjoint from each member of $\left\{A_{\alpha}: \alpha \in \beta\right\}$ then $\Phi$ restricted to $\mathscr{P}(D)$ 
is trivial. Even more is true. It is shown in pp. $145-150$ of [Sh] that there is a function $g^{0}$ with the following property: If $D$ is almost disjoint from each element of $\left\{A_{\alpha}: \alpha \in \beta\right\}$ then $D \subseteq^{*}$ domain $\left(g^{0}\right)$ and $\Phi(D) \equiv^{*} g^{0}(D)$. (The reader may worry that on p. 146 of [Sh] it is assumed that $A_{\alpha} \in T(\Phi)$ for $\alpha \in \omega_{1}$. This assumption, which will be crucial to $\S 3$, is not used however until p. 151 of [Sh].) It is easy to check, although not explicitly mentioned in $[\mathbf{S h}]$, that this property of $g^{0}$ implies that domain $\left(g^{0}\right) \equiv^{*} \omega \backslash \cup\left\{A_{\alpha}: \alpha \in F\right\}$ where $F$ is a finite subset of $\beta$.

Let $h_{n}$ be a function witnessing that $C_{n} \in T(\Phi)$. It now follows that if $h=$ $g^{0} \cup\left(\bigcup\left\{h_{n}: n \in F\right\}\right)$ then domain $(h) \supseteq^{*} C_{n}$ for $n \in \omega$ and, of course, domain $(h) \subseteq$ $T(\Phi)$. Hence $T(\Phi)$ is a $p$-ideal. The next section will show that PFA implies that $T(\Phi)$ is not a proper ideal. It is not known if this is necessary. In other words the following questions remain open.

QUESTION 2. Does the fact that $T(\Phi)$ contains an infinite set for every automorphism, $\Phi$, of $\mathscr{P}(\omega) /$ Finite imply that $T(\Phi)$ is a $p$-ideal for every automorphism?

QUESTION 3. Does the fact that $T(\Phi)$ is a $p$-ideal which contains an infinite set for every automorphism, $\Phi$, of $\mathscr{P}(\omega)$ /Finite imply that every automorphism is trivial?

3. It remains to be shown that PFA implies that every automorphism of $\mathscr{P}(\omega)$ /Finite is not only somewhere trivial but is actually trivial. The proof of this parallels that of Theorem 1 with one exception. Lemma 1 must be replaced by lemma 1a.

LEMMA 1a. In the extension by $\mathbf{P}$ there is a family $\left\{\left(A_{\alpha}, B_{\alpha}\right): \alpha \in \omega_{1}\right\}$ such that $\left\{A_{\alpha}: \alpha \in \omega_{1}\right\} \subseteq T(\Phi)$ is an almost disjoint family and there is a countable chain condition poset $\mathbf{Q}$ and a $\mathbf{Q}$-name $X$ such that

(12) $\mathbf{Q} \Vdash “ X \cap A_{\alpha} \equiv{ }^{*} B_{\alpha}$ ".

(13) $\mathbf{Q} \Vdash “(\forall Y \subseteq \omega)\left(\exists \beta \in \omega_{1}\right)(\forall \xi>\beta)\left(Y \cap \Phi^{*}\left(A_{\xi}\right) \not \equiv^{*} \Phi^{*}\left(B_{\xi}\right)\right)$.

Proof. A family $\left\{\left(A_{\alpha}, B_{\alpha}\right): \alpha \in \xi\right\}=\mathfrak{A}$ will be called very reasonable if it is reasonable and if $A_{\alpha} \in T(\Phi)$ for $\alpha \in \xi$. The proof of Lemma 1a now proceeds like that of Lemma 1 except that the filter $G$ on $\mathbf{D}$ must intersect more dense sets. The reason is that when $A_{\xi}=\bigcup\left\{C_{n} \backslash G(n): n \in \omega\right\}$ is constructed, care must be taken so that $\left\{\left(A_{\alpha}, B_{\alpha}\right): \alpha \in \xi+1\right\}$ is very reasonable-in particular $A_{\xi}$ must belong to $T(\Phi)$. Provided that each $C_{n}$ can be chosen in $T(\Phi)$, the result of $\S 2$ makes it possible to do this. Let $C \in T(\Phi)$ be an upper bound for each $C_{n}$. When $A_{\xi}$ is constructed with $\mathbf{D}$ it is only required to add countably many new dense sets to ensure that $A_{\xi} \subseteq C$ and hence is in $T(\Phi)$.

So it suffices to show that $C_{n}$ can be chosen in $T(\Phi)$. It is known from $\S 1$ that it is possible to define $\left(C_{n}, B_{n}\right)$ satisfying (7)-(9). Notice that if $C^{\prime} \subseteq C_{n}$ and $C^{\prime} \cap B_{n}$ is infinite then $\left(C^{\prime}, C^{\prime} \cap B_{n}\right)$ will also satisfy properties (7) and (9). In order to satisfy (8) it must be the case that for each $p \in \mathbf{Q}(\mathfrak{A})$ and $n \in \omega$ there is $m \geq n$ and $q \leq p$ such that $q$ forces that $m$ is a witness to the fact that $Y_{(\Psi(k))} \cap \Phi^{*}\left(C_{k}\right) \not \equiv^{*} \Phi^{*}\left(D_{k}\right)$. Let $W(p, n) \subseteq C_{n}$ be this set. Use Theorem 1 to find $W^{\prime}(p, n) \subseteq W(p, n)$ such that $W^{\prime}(p, n) \in T(\Phi)$ and $W^{\prime}(p, n) \cap B_{n}$ is infinite. Next use Theorem 2 to find $C \in T(\Phi)$ such that $C \supseteq^{*} W^{\prime}(p, n)$ for each $p$ and $n$. Then $C$ is as desired.

B. Velickovic has recently announced a negative answer to the following:

QUESTION 4. Does MA $\left(\aleph_{1}\right)$ imply that all automorphisms of $\mathscr{P}(\omega)$ /Finite are trivial? 


\section{REFERENCES}

[ARS] U. Abraham, M. Rubin, and S. Shelah, On the consistency of some partition theorems for continuous colorings and the structure of $\aleph_{1}$-dense real order types.

[Sh] S. Shelah, Proper forcing, Springer-Verlag, Berlin and New York, pp. 134-152.

Department of Mathematics, Rutgers University, New Brunswick, NeW JerSEY 08903

Department of MATHEMATICS, Hebrew UNiversity, JeRUSAlem, ISRAEl (Current address of Saharon Shelah)

Department of Mathematics, York University, North York, Ontario, CANADA M3J 1P3 (Current address of Juris Steprāns) 